\title{
El cambio climático, la salud humana y el desarrollo sostenible ${ }^{1}$
}

\author{
W. J. M. Martens ${ }^{2}$, R. Slooff ${ }^{3}$ y E. K. Jackson ${ }^{4}$
}

RESUMEN El cambio climático debido a actividades humanas pone en peligro los ecosistemas y la salud humana a escala mundial. Con el fin de hacer frente a las amenazas que se ciernen sobre los ecosistemas en todo el mundo, en el decenio de 1980 se introdujo el concepto del desarrollo sostenible. Desde entonces, ese concepto se ha aplicado ampliamente para guiar y enfocar la formulación de políticas. En el presente artículo se examinan las consecuencias sanitarias que tiene el cambio climático debido a actividades humanas para el desarrollo sostenible, en particular su posible efecto en el abastecimiento de alimentos, los desastres naturales, las enfermedades infecciosas, los ecosistemas y la elevación del nivel del mar. Se discute un modelo integrado que contiene los principales indicadores del desarrollo sostenible. También se examina la importancia que tienen el cambio climático, la salud humana y el desarrollo sostenible para las políticas internacionales pertinentes.

La salud de la población como "estado sostenible" (1) exige el continuo apoyo de actividades que permitan tener aire puro, agua limpia, suficientes alimentos, una temperatura tolerable, un clima estable, protección contra la radiación ultravioleta solar y altos grados de diversidad biológica. Los cambios socioeconómicos y las intervenciones sanitarias han mejorado la salud de la población en los últimos decenios, aunque todavía hay muchas disparidades en el estado potencial de

1 Se publicó en inglés en el Bulletin of the World Health Organization, Vol. 75, No. 6, 1997, con el titulo "Climate change, human health, and sustainable development". (C) Organización Mundial de la Salud, 1997.

2 Universidad de Maastricht, Maastricht, Países Bajos. Dirección postal: Universidad de Maastricht, Departamento de Matemáticas, P. O. Box 616, 6200 MD Maastricht, Países Bajos.

3 Consultor a corto plazo, Oficina del Programa Mundial e Integrado de Higiene del Medio, Organización Mundial de la Salud, Ginebra, Suiza.

4 Greenpeace International, Surry Hills, Sydney, Australia. salud alcanzado a escala mundial y sigue habiendo morbilidad enmendable y mortalidad prematura (2). No obstante, como efecto desfavorable del desarrollo económico, han comenzado a ocurrir cambios de salud resultantes del deterioro de las condiciones del medio ambiente mundial.

El Segundo Informe de Evaluación del Grupo Intergubernamental de Expertos sobre Cambios Climáticos (Intergovernmental Panel on Climate Change, IPCC) confirmó que las actividades humanas, sobre todo la quema de combustibles fósiles, han aumentado las concentraciones atmosféricas de gases importantes con efecto invernadero que pueden ocasionar el calentamiento de la superficie de la Tierra (3). También se determinó poco probable que el aumento de 0,3 a $0,6^{\circ} \mathrm{C}$ de la temperatura de la superficie del planeta observado en los últimos 100 años se deba a la variabilidad natural del sistema climático, y se llegó a la con- clusión de que el resto de las pruebas indican una clara influencia humana sobre el clima mundial.

El cambio climático debido a actividades humanas compromete ahora la sostenibilidad del desarrollo humano en el planeta porque amenaza los sistemas de apoyo ecológico de los que dependen la vida (4), la salud y el bienestar de la humanidad, cuya continua mejora debe ser la meta primordial del proceso de desarrollo propiamente dicho.

\section{El concepto del desarrollo sostenible}

El concepto del desarrollo sostenible se discutió por primera vez en 1980 en la publicación titulada World conservation strategy (5), pero solo logró amplia aceptación unos 10 años después. En 1987, en el informe titulado Our common future de la Comisión Mundial 
sobre el Medio Ambiente y el Desarrollo (6), se lanzó la siguiente definición del desarrollo sostenible que ha sido ampliamente citada: "El desarrollo que atiende a las necesidades de la generación actual sin poner en peligro las necesidades de las futuras generaciones". Esta definición antropocéntrica se basa en el principio de equidad entre las generaciones, en que se estipula que las futuras generaciones no deben heredar la carga de problemas ambientales evitables.

En esta reseña, el desarrollo sostenible se define desde un punto de vista más ecocéntrico: "Mejorar la calidad de la vida humana y, al mismo tiempo, vivir dentro de la capacidad máxima admisible de los ecosistemas de apoyo" (7). Esta definición tiene la ventaja de reconocer que el desarrollo es un proceso destinado a mejorar las condiciones de vida de la población y, al mismo tiempo, a reconocer que la capacidad del medio ambiente para apoyar esa iniciativa esfuerzo es limitada. De conformidad con ello, la Comisión de la OMS sobre la Salud y el Medio Ambiente declaró muy explícitamente que ninguna clase de desarrollo puede calificarse de sostenible si causa daño a la salud y al bienestar del ser humano (8).

El Convenio Marco de las Naciones Unidas sobre los Cambios Climáticos tiene por objetivo primordial (Artículo 2) la estabilización de las concentraciones de gases con efecto invernadero a un nivel que prevenga los efectos "peligrosos" para el sistema climático, y exige concretamente que ello se logre dentro de un marco cronológico suficiente ". . . para asegurarse de que la producción de alimentos no se vea amenazada y permitir que el desarrollo económico prosiga de una forma sostenible". Además, los principios del Convenio (Artículo 3) exigen específicamente que al lograr ese objetivo se tenga en cuenta a los países con particular vulnerabilidad a los efectos adversos del cambio climático. En los principios del Convenio también se reconoce el derecho que tiene cada país a lograr un desarrollo sostenible.

\section{El cambio climático y la salud humana: ¿desarrollo sostenible?}

Todos los sistemas ecológicos y socioeconómicos indispensables para el desarrollo y bienestar del ser humano, incluso para la salud, son sensibles a la velocidad y magnitud del cambio climático (9). Un Grupo de Trabajo Interinstitucional establecido para evaluar los posibles efectos sanitarios del cambio climático (10) reconoció que esa evaluación debe hacerse contra el telón de fondo de muchos cambios ambientales a escala mundial, no todos relacionados con el clima. King ha señalado que varios países en desarrollo están "atrapados en sentido demográfico" porque las comunidades han excedido o posiblemente excederán la capacidad máxima admisible de sus ecosistemas locales, su capacidad para emigrar y la capacidad de la economía para producir bienes y servicios a cambio de alimentos y artículos de primera necesidad (1). Esos fracasos del desarrollo sostenible ocurren independientemente del cambio climático y están marcados por los patrones de salud relacionados con las enfermedades infecciosas, la malnutrición y el hambre (aliviada en parte con ayuda alimentaria) y los actos genocidas de agresión en masa.

El cambio climático podría infligir más estrés a ecosistemas ya sobrecargados. En el plano mundial, el Grupo de Trabajo Interinstitucional prevé que algunos de los efectos más graves del aumento de temperatura y del cambio de la precipitación, la mayor frecuencia de acontecimientos climáticos extremos y la elevación del nivel del mar en todo el mundo podrían recaer sobre algunos de los países menos desarrollados. Además, se prevé que el hambre específica de cada región aumentará mucho en cualquiera de los marcos hipotéticos del clima mundial aceptados por el IPCC, aun si se deja campo para considerar el efecto del crecimiento demográfico propiamente dicho. En la actualidad, alrededor de 530 millones de personas están expuestas anualmente al riesgo de sufrir hambre: al llegar el año 2060 podría haber otros 40 a 300 millones (en relación con una población estimada de 640 millones de personas expuestas al riesgo de hambre para ese entonces, sin los efectos del cambio climático) por causa de varios factores, incluso del crecimiento demográfico. Esta expectativa, que puede ser optimista (11), se basa en proyecciones de los cambios del potencial de producción agrícola de los principales cultivos básicos y los cambios resultantes de la productividad en cada uno de los marcos hipotéticos del cambio climático aceptados por el IPCC (12).

Alrededor de 250000 personas mueren cada año como consecuencia de desastres naturales y cerca de $95 \%$ de esas defunciones ocurren en los países pobres. Esta distribución es quizá un buen punto de reflexión sobre las diferencias en el grado de mitigación de los efectos de los desastres y de preparación para situaciones de esa índole que existen entre los países ricos y pobres. Por ende, no es difícil prever dónde se producirá la mayoría del daño adicional si llegan a ocurrir cambios climáticos extremos con más frecuencia o cuando la elevación del nivel del mar destruya más zonas costeras. Puesto que varios de los países más vulnerables a los efectos de los desastres naturales (India, Bangladesh y África subsahariana) ya están superpoblados, no hay campo para mitigar esos efectos con evacuación obligada o planificación del uso restringido de la tierra.

La elevación del nivel del mar, las inundaciones y la erosión del litoral tendrán un efecto directo en la viabilidad de muchas poblaciones costeras. Las estimaciones indican que alrededor de 46 millones de personas están expuestas actualmente al riesgo de mareas de tormenta. Sin tener en cuenta el crecimiento demográfico previsto y las medidas de adaptación, una elevación de $50 \mathrm{~cm}$ del nivel del mar (escala proyectada actualmente: de 15 a $115 \mathrm{~cm}$ en el siglo venidero) aumentaría esa cifra a 92 millones de personas (9). Esa elevación del nivel del mar podría amenazar a culturas enteras y forzar a las poblaciones a una emigración interna o internacional (13). 
Se prevén cambios en la distribución de las enfermedades infecciosas y una mayor frecuencia de infecciones nuevas y reemergentes en todos los marcos hipotéticos de cambio climático aceptados por el IPCC. Por ejemplo, podría aumentar la carga mundial neta de la malaria (población actualmente expuesta a riesgo: $2,4 \times 10^{9}$ ), aunque la menor humedad del suelo reduzca su incidencia en algunas zonas. Una estimación indica que otros 100 a 700 millones de personas podrían estar expuestas al riesgo de malaria causada por el cambio climático debido a actividades humanas en el año $2050(14,15)$ y que otros $1000 \mathrm{mi}-$ llones podrían estar infectados al llegar el año 2100.

Los ecosistemas naturales proporcionan muchos bienes y servicios importantes para el desarrollo sostenible, entre ellos los siguientes: alimentos, fibra, medicinas y energía; absorción y conservación de carbono y otros nutrientes; asimilación de desechos, purificación del agua, regulación de la escorrentía y control de las inundaciones, la degradación del suelo y la erosión de las playas; y actividades de recreo y turismo (9). El cambio climático puede alterar la composición de muchos ecosistemas y reducir la diversidad biológica y los servicios que proporcionan los ecosistemas (9). Es posible que algunos ecosistemas forestales y montañosos desaparezcan del todo y que la desertificación se agrave y no sea tan fácil de corregir. Los ecosistemas acuáticos y marinos importantes para el turismo, las reservas de agua dulce, la pesca y la diversidad biológica, como las zonas húmedas costeras, los arrecifes de coral y los deltas fluviales, estarán expuestos a un mayor riesgo por el cambio climático y la elevación del nivel del mar.

La materialización de las posibles repercusiones del cambio climático para la salud pública dependerá de las suposiciones hechas, la idoneidad de los modelos usados y el grado de mitigación y adaptación factible, aceptable y económicamente asequible. Es de esperar que un país con una producción agrícola en deterioro, pero con su- ficiente potencial de crecimiento industrial, alimente a su población con bienes importados. Un país exento de malaria con un sistema de salud pública en buen funcionamiento puede frenar el peligro de la malaria sin siquiera aumentar el gasto del sector de la salud. Sin embargo, los efectos del cambio climático pueden ser acumulativos para las poblaciones vulnerables: la malnutrición puede exacerbar la enfermedad y la muerte ocasionadas por enfermedades infecciosas, algunas de las cuales contribuyen a la malnutrición. El cambio climático antropógeno puede influir en la malnutrición y la infección.

\section{Indicadores de sostenibilidad}

¿Cómo se determina la "sostenibilidad"? ¿Cuáles son los indicadores del desarrollo sostenible (un indicador se define como una característica del estado y del comportamiento de un sistema dinámico) y cuáles son las metas y los valores de referencia de esos indicadores?

En los índices de salud convencionales, como las tasas de esperanza de vida y de mortalidad, no se tiene en cuenta que las bases de la salud humana dependen de la integridad y productividad de los procesos sustentadores de la vida de un ecosistema (particularmente de los relacionados con la estabilidad del clima y la producción de alimentos) (4). La salud de las poblaciones humanas es uno de los componentes de la compleja interacción que ocurre entre los procesos demográficos, biológicos, ecológicos, sociales y económicos. En el experimento modelo descrito a continuación, que se basa en un análisis de los efectos del cambio climático en la malaria $(16,17)$, presentamos un sencillo ejemplo de cómo se pueden evaluar los indicadores económicos, ecológicos y sanitarios simultáneamente dentro de un mismo marco.

El cambio de temperatura se puede considerar como un indicador sencillo de riesgo ecológico: una tasa general de cambio de temperatura de $0,1{ }^{\circ} \mathrm{C}$ por decenio es el límite máximo en exceso del cual se prevé que aumentarán rápidamente los riesgos de grave daño a los ecosistemas y a las zonas costeras vulnerables y de cambios repentinos del sistema climático (18). El proceso conducente al efecto de un cambio climático debido a actividades humanas en la incidencia de malaria puede representarse por los posibles cambios de la carga de morbilidad causada por la malaria. En condiciones ideales, la carga de la morbilidad provocada por la malaria debe ser de cero, aunque quizá nunca se alcance ese nivel. Para fines de la salud, la meta aquí propuesta es, por tanto, un cambio de cero en la carga de la morbilidad (expresada como el número de años de vida perdidos ajustados en función de la discapacidad) (AVAD) (19). Esto implica que cualquier aumento de la carga de la morbilidad se considera insostenible. El volumen de recursos asignados a servicios de salud puede servir de amortiguador contra los efectos adversos del cambio climático mundial para la salud. La dinámica y el grado de desarrollo económico, expresados como el crecimiento del producto interno bruto (PIB) per cápita, pueden considerarse indicadores de los medios a disposición del sector de la salud pública (20).

La figura 1 muestra los cambios de la carga de la morbilidad causada por la malaria, la velocidad con que cambia la temperatura y el desarrollo económico según los tres marcos hipotéticos aceptados por el IPCC (IS92a, IS92c e IS92e). Las máximas emisiones de gases con efecto invernadero provienen del marco hipotético IS92e, en que se juntan, entre otras suposiciones, una tasa moderada de crecimiento demográfico, un elevado crecimiento económico y una abundancia de combustibles fósiles. Las emisiones más pequeñas de gases con efecto invernadero provienen del marco hipotético IS92c, en que se presupone que la población crecerá y luego disminuirá a mediados del siglo venidero, que el crecimiento económico es bajo y que las existencias de combustibles fósiles son sumamente limitadas (21). 
FIGURA 1. Tres marcos hipotéticos de 1992 aceptados por el IPCC con respecto a indicadores de salud, ecológicos y económicos (IS92a, IS92c e IS92e). El indicador de salud se expresa como el exceso de años de vida perdidos ajustados en función de la discapacidad (AVAD) por 1000 habitantes, en relación con el marco hipotético de referencia (sin ningún cambio de temperatura); el indicador ecológico como el aumento relativo de temperatura ( ${ }^{\circ} \mathrm{C}$ por decenio); y el indicador económico como la diferencia entre el crecimiento del PIB per cápita (\% anual) y el crecimiento de base (1955-89; $2 \%$ anual). Los tres indicadores se trazan en un plano bidimensional. En el caso del desarrollo sostenible, los cambios de los indicadores de salud, ecológicos y económicos no deben ir más allá del plano abarcado por los valores escogidos como objetivo (es decir, los límites del sistema). Es importante señalar que eso depende mucho de los indicadores y valores de referencia seleccionados $y$ de las suposiciones y la incertidumbre que encierra el experimento de modelación.

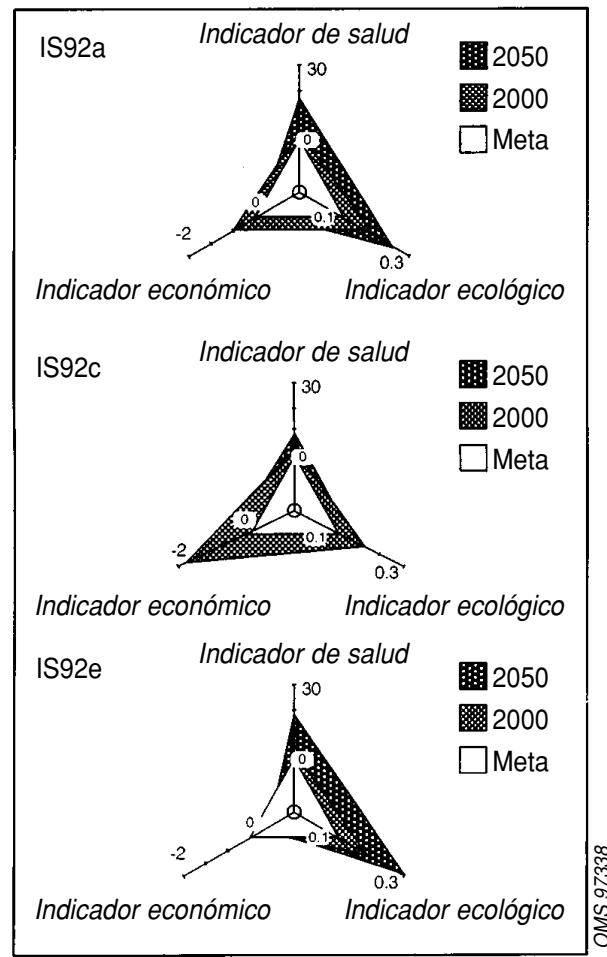

La meta de un aumento de la temperatura media mundial relativa de $0,1{ }^{\circ} \mathrm{C}$ por decenio se supera en todo el período de simulación (1990-2050) en los tres marcos hipotéticos emplea- dos. La meta de un aumento nulo del número de años de vida sana perdidos por causa de malaria no se alcanza con ninguno de los cálculos y el mayor número de años de vida sana se pierden en el marco hipotético IS92e. Las proyecciones de crecimiento anual del PIB per cápita en el período 1955-1989 en las regiones maláricas varió de 1,0\% en África subsahariana a 4,6\% en Asia. Las presuposiciones de crecimiento económico en los marcos hipotéticos IS92a e IS92c son inferiores a las tasas históricas; el marco hipotético IS92e muestra un aumento del crecimiento económico. Sin embargo, puesto que las opciones de mitigación y adaptación que tienen los países dependen, por lo menos en parte, de la trayectoria de desarrollo de su economía que ya compromete la sostenibilidad (como en el caso de la mayoría de los países pobres), la carga de la morbilidad provocada por el cambio climático quedará sin mitigar o se mitigará a un alto costo para la economía. En cualquier caso, la sostenibilidad del desarrollo correrá mayor peligro del que habría tenido sin el cambio climático debido a actividades humanas.

\section{Protección de la salud de la población}

Los planes para proteger la salud humana contra los posibles efectos del cambio climático mundial (y los efectos relacionados que tienen para la salud humana las mayores concentraciones de radiación ultravioleta como resultado del agotamiento de la capa de ozono y la contaminación del aire de las zonas urbanas, tema no discutido aquí con detalles) exigen que se entiendan mucho mejor los mecanismos desencadenantes de la enfermedad en cada caso, los posibles efectos sinérgicos y la vulnerabilidad de las poblaciones. Es indispensable establecer indicadores válidos para los cambios en las condiciones de salud ambiental, en relación con los experimentos basados en el uso de modelos (como se discute en el presente artículo) y con la vigilancia de los indicadores de salud ambien- tal en regiones vulnerables. Por ejemplo, la vigilancia de la propagación de los mosquitos causantes de la malaria o de otros vectores a lugares actualmente exentos de las enfermedades que acarrean y la mejora de los sistemas de alerta anticipada sobre condiciones meteorológicas extremas y desastres naturales ayudarán a poner en práctica las medidas de preparación para situaciones de emergencia. El perfeccionamiento de los sistemas de pronóstico del clima y su aplicación a la planificación agrícola podrían ayudar a mejorar la seguridad alimentaria y a evitar el hambre generalizada como consecuencia de la pérdida de cultivos.

Los campos de investigación que conviene estimular a mediano plazo incluyen los siguientes: la elaboración de mejores modelos integrados para evaluar los riesgos que acarrean los ecosistemas para la salud humana a causa de la variabilidad del clima; la relativa importancia de las adaptaciones técnicas, como el acondicionamiento de aire y otras respuestas de adaptación a las variaciones extremas del clima; y la interacción del aumento de la temperatura con los mayores niveles de radiación ultravioleta y la calidad del aire exterior (particularmente en las zonas urbanas). En el plano nacional, convendría instar a los países a hacer una evaluación general de la vulnerabilidad de la población a los posibles efectos sanitarios de los diversos componentes del cambio climático, incluso un aumento de la radiación ultravioleta y de la contaminación del aire urbano.

Si los efectos para la salud humana que tiene el cambio climático en el largo plazo son tan graves como se indica, la base sostenida más eficaz para la mitigación consiste en reducir la emisión de gases con efecto invernadero (9). El sector de la salud puede desempeñar una función activa al asegurarse de que los gobiernos asignen prioridad a estrategias que reduzcan notablemente las emisiones de anhídrido carbónico, concentrándose sobre todo en medidas de uso eficiente de energía y en el aprovechamiento y uso de fuentes de energía renovables (22). 
Casi todas las evaluaciones del efecto del cambio climático se basan en marcos hipotéticos en que la concentración atmosférica de anhídrido carbónico es el doble de la registrada en la época preindustrial. Los posibles efectos sanitarios del cambio climático y el principio de precaución ofrecen argumentos racionales para estabilizar la concentración atmosférica en un nivel inferior al citado. También se necesita una transferencia de tecnologías de alto rendimiento energético para no desviar el desarrollo económico a corto plazo ni comprometer la atención de las necesidades de salud de la generación actual.

\section{CONCLUSIONES}

Esta breve reseña demuestra que el debate sobre los efectos sanitarios de los cambios climáticos y el desarrollo sostenible debe continuar a escala mundial y nacional, dentro de un marco integrado global. Cada país debe evaluar primero su actual situación de salud en función de factores de desarrollo económico y clima, y posteriormente su vulnerabilidad a los efectos sanitarios del cambio climático y sus posibilidades de mitigación y adaptación. Ulteriormente, es preciso adaptar las políticas en materia de población, agricultura e industria a metas ecológicamente aceptables en lo que respecta al bienestar de la población y a los efectos económicos para otros países, y someterlas a rigurosos criterios de sostenibilidad.

Aunque sigue habiendo incertidumbre en lo que respecta a la evaluación de lo que constituye un cambio climático "peligroso", los efectos proyectados del cambio climático para la salud internacional son una clara amenaza para el desarrollo sostenible, especialmente en las naciones más pobres del mundo y, por ende, ofrecen un argumento convincente para acelerar las negociaciones y medidas en marcha a fin de reducir las emisiones de gases con efecto invernadero. Como señala el IPCC: "Si se emplean con cuidado, esas respuestas ayudarían a enfrentar la dificultad del cambio climático y a mejorar las perspectivas de desarrollo económico sostenible de todos los pueblos y naciones".

Agradecimiento. Los autores expresan su agradecimiento a Maurice King, Tony McMichael y Barrie Pittock por sus valiosas observaciones sobre el manuscrito.

\section{REFERENCIAS}

1. King M. Health is a sustainable state. Lancet 1990;336:664-667.

2. World health report 1995. Geneva: World Health Organization; 1995.

3. Intergovernmental Panel on Climate Change. En: Houghton JJ, et al., eds. Climate change 1995: The science of climate change. New York: Cambridge University Press; 1996.

4. McMichael AJ. Planetary overload: Global environmental change and the health of the human species. Cambridge, MA: Cambridge University Press; 1993.

5. World conservation strategy: Living resource conservation for sustainable development. Gland, Switzerland: International Union for Conservation of Nature and Natural Resources (IUNC) United Nations Environment Programme/World Wildlife Fund (WWF); 1980.

6. World Commission on Environment and Development. Our common future. Oxford: Oxford University Press; 1987.

7. Caring for the Earth: A strategy for sustainable living. Gland, Switzerland: International Union for Conservation of Nature and Natural Resources (IUNC) United Nations Environment Programme (UNEP)/World Wildlife Fund (WWF); 1991.

8. Our planet, our health. Geneva: World Health Organization; 1992.
9. Intergovernmental Panel on Climate Change. En: Watson RT, et al., eds. Climate change 1995 Impacts, adaptations, and mitigation of climate change: Scientific-technical analyses. New York: Cambridge University Press; 1996.

10. McMichael AJ, et al., eds. Climate change and human health: An assessment prepared by a Task Group on Behalf of the World Health Organization, the World Meteorological Organization and the United Nations Environment Programme. (Documento inédito WHO/EHG/ 96.7).

11. Pittock AB, Whetton P, Wang Y. Climate change and food supply. Nature 1994;371:25.

12. Parry ML, Rosenzweig C. Food supply and risk of hunger. En: Health and climate change. London: Lancet; 1993.

13. Intergovernmental Panel on Climate Change. IPCC second assessment synthesis of scientifictechnical information relevant to interpreting Article 2 of the UN framework convention on climate change. Geneva: Intergovernmental Panel on Climate Change (IPCC)/World Meteorological Organization (WMO)/United Nations Environment Programme (UNEP).

14. Martens WJM, et al. Climate change and vector-borne diseases: A global modelling perspective. Global environmental change 1995; 5(3):195-209.
15. Martens WJM. Health impacts of climate change and ozone depletion: An eco-epidemiological modelling approach. [Tesis doctoral]. Maastricht, Países Bajos: Maastricht University; 1997.

16. Martens WJM, et al. Potential impact of global climate change on malaria risk. Environmental health perspectives 1995;103:458-464.

17. Martens WJM. Global atmospheric changes and human health: An integrated modelling approach. Climate research 1996;6:107-112.

18. Targets and indicators of climatic change. En: Rijsberman FR, Swart RJ, eds. Report of Working Group II of the Advisory Group on Greenhouse Gases (AGGG). Stockholm: Stockholm Environmental Institute; 1990.

19. Murray CJL. Quantifying the burden of disease: The technical basis for disabilityadjusted life years. Bull World Health Organ 1994;72:429-445.

20. World Health Organization. The malaria situation in 1973. Chron World Health Organ 1974; 28:479-487.

21. Intergovernmental Panel on Climate Change. Climate change 1992: The supplementary report to the IPCC scientific assessment. New York: Cambridge University Press; 1992.

22. Jackson EK. Climate change and global infectious disease threats. Med J Australia 1995;163: 570-574. 
ABSTRACT Human-induced climate change threatens ecosystems and human health on a global scale. In order to withstand the worldwide threats to ecosystems, the concept of sustainable development was introduced during the 1980s. Since then, this concept has

\section{Climate change, human health, and sustainable development}

been widely applied to guide and focus policy-making. The present article reviews the health consequences of human-induced climate change on sustainable development, particularly the potential impact of such change on food supply, natural disasters, infectious diseases, ecosystems, and sea level rise. Discussed is an integrated model containing the key indicators of sustainable development. The relevance of climate change, human health, and sustainable development for international climate change policy is also examined.

\section{Ganadores del Premio Abraham Horwitz en Salud Interamericana, 1998}

La Fundación Panamericana de la Salud y Educación (PAHEF) se complace en participar que, conforme a las recomendaciones del Comité del Premio, la Junta de Directores concederá el Premio Abraham Horwitz en Salud Interamericana para 1998 al matrimonio hondureño doctor Carlos Ponce y señora Elisa de Ponce. Esta pareja de científicos ha trabajado desde hace años para mejorar los conocimientos, la prevención, el control, el tratamiento y el manejo de la enfermedad de Chagas y las leishmaniasis en Centroamérica y otras partes de la Región.

El Premio, que honra la larga y fructífera carrera del doctor Abraham Horwitz, Director Emérito de la OPS, está dedicado a estimular la excelencia y el liderazgo en el campo de la salud pública de las Américas. Este se pondrá en manos del matrimonio Ponce en septiembre de 1998, durante la XXV Conferencia Sanitaria Panamericana. La Revista Panamericana de Salud Pública/Pan American Journal of Public Health les ofrece sus parabienes. 